\title{
ON THE FUNDAMENTAL DOMAIN OF A DISCRETE GROUP
}

\author{
MELVYN B. NATHANSON
}

\begin{abstract}
Gel'fand, Graev, and Pjateckiï-Šapiro proposed a method to construct the fundamental domain of a discrete group acting on certain metric spaces. A counterexample is given to show that this construction sometimes fails.
\end{abstract}

Let $W$ be a topological space, and let $\Gamma$ be a discrete group of homeomorphisms of $W$. The group $\Gamma$ is discrete if for every $w \in W$ the set of points $\gamma w$ with $\gamma \in \Gamma$ has no limit point in the space $W$. A fundamental domain of the group $\Gamma$ in $W$ is an open set $F \subset W$ such that (1) if $\gamma_{1}$, $\gamma_{2} \in \Gamma$ and $\gamma_{1} \neq \gamma_{2}$, then $\gamma_{1} F \cap \gamma_{2} \bar{F}=\varnothing$, and (2) $W$ is the union of the sets $\gamma \bar{F}$ with $\gamma \in \Gamma$.

Suppose that $W$ is a locally compact metric space, that the metric $d$ on $W$ has the "convexity" property that for every $w_{0}, w_{1} \in W$ there exists $w_{2} \in W$ such that $d\left(w_{0}, w_{2}\right)=d\left(w_{1}, w_{2}\right)=\frac{1}{2} d\left(w_{0}, w_{1}\right)$, and that $d\left(w_{0}, w_{1}\right)=$ $d\left(\gamma w_{0}, \gamma w_{1}\right)$ for every $\gamma \in \Gamma$. Choose a point $w_{0} \in W$ such that $\gamma w_{0} \neq w_{0}$ for all $\gamma \in \Gamma$ with $\gamma \neq e$. Define

$$
\begin{aligned}
& A=\left\{w \in W \mid d\left(w, w_{0}\right)<d\left(w, \gamma w_{0}\right) \text { for all } \gamma \in \Gamma, \gamma \neq e\right\}, \\
& B=\left\{w \in W \mid d\left(w, w_{0}\right) \leqq d\left(w, \gamma w_{0}\right) \text { for all } \gamma \in \Gamma\right\} .
\end{aligned}
$$

In [1, p. 6] it is claimed that $\bar{A}=B$ and that $A$ is a fundamental domain of $\Gamma$ in $W$. But the following example shows that both these claims are false. (If indeed $\bar{A}=B$ and if also every closed and bounded set in $W$ is compact, then the argument in [1] does prove that $A$ is a fundamental domain of $\Gamma$ in $W$.)

Take the plane $R^{2}$ and define the distance between two points by

$$
d\left(\left(x_{1}, y_{1}\right),\left(x_{2}, y_{2}\right)\right)=\left|x_{1}-x_{2}\right|+\left|y_{1}-y_{2}\right| \text {. }
$$

This metric is equivalent to the usual metric on the plane. Let $\Gamma=$ $\left\{\gamma_{n} \mid n=0, \pm 1, \pm 2, \cdots\right\}$ be the discrete group of translations $\gamma_{n}(x, y)=$ $(x+n, y+n)$. Then $\gamma_{n}(0,0)=(n, n) \neq(0,0)$ for all $n \neq 0$, and

$$
A=\{(x, y)|| x|+| y|<| x-n|+| y-n \mid \text { for all } n \neq 0\} .
$$

Received by the editors January 31, 1973 and, in revised form, April 3, 1973. AMS (MOS) subject classifications (1970). Primary 57E30; Secondary 57E25.

Key words and phrases. Fundamental domain, discrete group. 
For $n \neq 0$, let $A_{n}=\{(x, y)|| x|+| y|<| x-n|+| y-n \mid\}$. If $n>0$, then $A_{n}=$ $\{(x, y) \mid x<n$ and $y<\min (n, n-x)\}$ and $A_{-n}=\{(x, y) \mid x>-n$ and $y>$ $\max (-n,-n-x)\}$. Then $A_{n} \cap A_{-n}$ is the open hexagon

$$
\{(x, y) \mid-n<x<n \text { and } \max (-n,-n-x)<y<\min (n, n-x)\} \text {. }
$$

Therefore, $A=\bigcap_{n=1}^{\infty}\left(A_{n} \cap A_{-n}\right)$ is simply the open hexagon

$A=\{(x, y) \mid-1<x<1$ and $\max (-1,-1-x)<y<\min (1,1-x)\}$.

Similarly,

$$
B=\{(x, y)|| x|+| y|\leqq| x-n|+| y-n \mid \text { for all } n\}
$$

is the closed hexagon $\bar{A}$ together with the entire second and fourth quadrants of the plane. Clearly, $\bar{A} \neq B$ and $A$ is not a fundamental domain of $\Gamma$ in $R^{2}$. Note that the infinite strip $F=\{(x, y) \mid-1-x<y<1-x\}$ is a fundamental domain, and that $A \subset F \subset F \subset B$.

\section{REFERENCE}

1. I. M. Gel'fand, M. I. Graev and I. I. Pjateckii-Šapiro, Generalized functions. Vol. 6: Theory of representations and automorphic functions, "Nauka", Moscow, 1966; English transl., Saunders, Philadelphia, Pa., 1969. MR 36 \#3725; 38 \#2093.

Department of Mathematics, Southern Illinois University, Carbondale, ILLINOIS 62901 